\title{
Optimal features for online seizure detection
}

\author{
Lojini Logesparan · Alexander J. Casson · Esther Rodriguez-Villegas \\ Electrical and Electronic Engineering Department, Imperial College London, \\ SW7 2AZ, UK \\ Tel.: +44 (0)20 75946297 \\ Fax: +44 (0)20 75814419 \\ E-mail: lojini.logesparan04@imperial.ac.uk
}

\begin{abstract}
This study identifies characteristic features in scalp EEG that simultaneously give the best discrimination between epileptic seizures and background EEG in minimally pre-processed scalp data; and have minimal computational complexity to be suitable for on-line, real-time analysis. The discriminative performance of 65 previously reported features has been evaluated in terms of sensitivity, specificity, Area Under the sensitivity-specificity Curve (AUC), and relative computational complexity, on 47 seizures (split in 26982 s sections) in over 172 hours of scalp EEG from 24 adults. The best performing features are line length and relative power in the 12.5$25 \mathrm{~Hz}$ band. Relative power has a better seizure detection performance ( $\mathrm{AUC}=0.83$; line length AUC $=0.77$ ), but is calculated after the Discrete Wavelet Transform and is thus more computationally complex. Hence, relative power achieves the best performance for offline detection, whilst line length would be preferable for online low complexity detection. These results, from the largest systematic study of seizure detection features, aid future researchers in selecting an optimal set of features when designing algorithms for both standard offline detection and new online low computational complexity detectors.
\end{abstract}

Keywords Seizure detection $\bullet$ Feature $\bullet$ Online $\bullet$ EEG $\bullet$ Epilepsy 


\section{Introduction}

Epilepsy is a serious neurological disorder that affects 50 million people worldwide [28]. Electroencephalography (EEG) is a key tool for the diagnosis and treatment of the disorder and the benefits of long-term EEG monitoring, for both diagnosis and treatment, have been extensively reported $[3,21,28]$. However, EEG monitoring over many days, or even weeks, generates long records that are cumbersome for neurologists to reviewvisual inspection takes up to two hours per 24 hour recording [9].To alleviate this workload, seizure detection algorithms to automatically detect the presence of epileptiform activity have long been of research interest. Seizure detection algorithms are also of use to alert medical practitioners or bystanders of the occurrence of a clinical or sub-clinical seizure, and recently there has been renewed interest in discontinuous EEG analysis [2,3]. Here, seizure detection algorithms aim to mark-out epileptiform activity so that the neurologist only analyses these marked sections of EEG rather than the complete EEG trace. Such sampled reviews have now been reported to capture sufficient information such that the final electro-clinical diagnosis is in close agreement with the diagnosis resulting from the review of the full continuous recording $[2,18]$. However, despite the clear need for seizure detection algorithms, current algorithms still do not give sufficiently accurate results to be of practical use to clinicians and patients $[16,27,34]$. To improve the performance of seizure detection algorithms many researchers $[10,11$, $19,20,26,29,30]$ have compared different characteristic features of seizure and nonseizure EEG sections to determine which features can be used to best separate ictal and interictal EEG. These then facilitate designing future algorithms with the optimal set of features, in order to achieve better seizure detection performance. However, these comparison studies only evaluate the utility of different characteristic features in terms of their performance for separating ictal and interictal activity: in terms of sensitivity, specificity, false positive rate, and the area under the performance trade-off curve. In recent years there has also been significant interest in the development of miniaturised, portable EEG systems for prolonged ambulatory monitoring that incorporate online, realtime, seizure detection within the ambulatory unit $[3,12,22,26,32]$. This could be used to alert medical practitioners or bystanders of the occurrence of a clinical or sub-clinical seizure, to facilitate closed loop treatment [13], or to collect discontinuous data for sampled review. In these seizure detection systems the computational complexity required to generate each feature is an essential comparison point.

Traditionally, when an algorithm is implemented offline using a standard computer, algorithms with higher computational complexity can be expected to have longer simulation times, but this is not a critical factor using modern systems. On the other hand, 
in battery powered ambulatory EEG systems, computationally complex algorithms can be expected to have higher power consumption due to the increased number of processing stages (additions, multiplications and similar) required. This translates to shorter monitoring times caused by a shorter lifetime for the battery, or the requirement for obtrusive physically larger batteries. Thus for future ambulatory seizure detection systems it is essential when evaluating features, to consider not only the detection accuracy (sensitivity and specificity) of each feature, but also the computational complexity. A recent study [26] investigated six features on invasive EEG recordings of animal models and gave some insight to the two-way trade-off of detection accuracy and hardware cost (including power consumption), for a specific hardware architecture. This paper presents an investigation in to the performance of 65 features in terms of both detection performance and computational complexity, when used for seizure detection in minimally pre-processed adult scalp EEG. The 65 features utilised have been taken from 97 publications considering scalp EEG seizure detection published between 2000 and 2010. A simple seizure detection algorithm has been developed to compare the performance of each feature in turn on 172 hours of scalp EEG data containing 47 seizure events. It should be stressed that the seizure detection algorithm described here has not been developed for optimal detection accuracy, and thus incorporating extra processing, for example to remove artefacts or interictal spikes that may occur in the scalp EEG, could improve the algorithm performance. Instead, the aim is to consider the utility of each individual feature to facilitate future algorithms which would incorporate such extra processing stages. In addition to measuring standard seizure detection performance (using sensitivity and specificity), the computational complexity has been measured as a relative value using the simulation time required to generate each feature in MATLAB. This avoids specific architecture dependent computational complexity results and the increased simulation time for higher computational complexity features allows comparative results to be generated on a standard computer.

\section{Methods}

To determine suitable features to compare, a systematic review of recent seizure detection algorithms for scalp EEG-based monitoring, and published since the last such review [9], was performed. The inclusion criteria for publications considered in the review and full details of the 97 short-listed publications is given in the supplementary material. Based on this review, 65 features derived from: the time domain EEG signal, the Discrete Wavelet Transform (DWT) of the time domain EEG signal, the Continuous Wavelet Transform 
(CWT) of the EEG and the Fourier Transform (FT) of the EEG, were selected. These preprocessing techniques are particularly suitable for algorithms to be designed for online long-term monitoring from battery-powered systems, as features derived from the time domain signal do not require any additional processing and the other three pre-processing techniques have previously been implemented in low power dedicated circuits: DWT [15, 23], CWT [1, 4, 14] and FT [33].

\subsection{Features analysed}

The features selected for comparison from the 97 shortlisted publications have been summarised in Table 1 and the number of publications that utilise each of these features is also listed. The core features are categorized in to: 17 time domain, 8 DWT based, 4 CWT based and 6 FT based features. There are 35 core features present, and then the DWT based features and 2 FT based features (power and spectral entropy) are calculated multiple times, based upon using different frequency bands from the time-frequency transformation. In this study, well-established frequency bands for epileptiform activity $(0-25 \mathrm{~Hz}[8])$ have been selected, which at the same time reduce signal disruptions due to high frequency artefacts $[7,8,25]$. For the DWT, these frequency bands result from a standard five scale decomposition of the input EEG signal and span: D3 (12.5-25Hz); D4 $(6.25-12.5 \mathrm{~Hz})$; D5 $(3.125-6.25 \mathrm{~Hz})$; and A5 $(0.16-3.125 \mathrm{~Hz})$.The same frequency bands were selected for the two FT features. Details on the full calculation for each feature and the implementation of the respective pre-processing methods can be found in the supplementary material.

\subsection{Comparison methods}

To investigate the performance of each individual feature listed in Table 1, each feature is tested in turn using the simple seizure detection algorithm illustrated in Fig. 1.

The core operation of this algorithm is as follows. First, the input EEG discrete-time signal (resampled at $200 \mathrm{~Hz}$ ) is split into non-overlapping $2 \mathrm{~s}$ epochs $e$ and the feature $F(e)$ calculated for each epoch. To distinguish between seizure and non-seizure epochs based upon $F(e)$, a fixed threshold $\beta$ is used. If $F(e)>\beta$, the epoch is marked as ictal, otherwise it is interictal and this provides the output to be compared to expert markings present in prerecorded EEG signals.(Note that for features that are expected to reduce during a seizure, a detection will occur if the normalized feature falls below $\beta$.) A simple threshold allows the performance of each feature to be investigated in turn and by running the algorithm multiple times with different values for the detection threshold $\beta$, the trade- 
off between correct detections and incorrect detections for the feature can also be investigated.

As the aim here is to investigate the performance of each feature in turn, the signal conditioning and post-processing used are kept to a minimum. From Fig. 1, the recorded EEG data is high pass filtered with a cut-off frequency of $0.16 \mathrm{~Hz}$ to ensure all data is inline with the International Federation of Clinical Neurophysiology recommendation [24]. For post-processing, prior to applying the threshold, the calculated feature in each epoch $F(e)$ is normalized to restrict its value between $(0,1)$ and correct for different amplitudes of the input EEG data. This normalization is done using a peak detector to find the maximum value of the feature $F(e)$ over time and using this value to normalize $F(e)$. This method has two main advantages: firstly, the normalization does not use the raw EEG signal, reducing any errors caused by high amplitude artefacts; and secondly, as the maximum is calculated over time the normalized feature will never exceed one. At the start of each record, the epoch value $e$ equals 1 , the peak value $z(e)$ is set to $F(e)$, and only updated if any subsequent value of $F(e)$ exceeds $z(e-1)$. The normalized feature $N(e)$ is then calculated as $N(e)=F(e) / z(e)$ and compared to the detection threshold $\beta$. In cases where $F(e)$ is always negative, it is multiplied by -1 prior to normalization.

To generate the final detection decision, the algorithm in Fig. 1 is applied to each channel of EEG data separately. The output of the algorithm is a vector of non-overlapping epochs classified as either seizure (marked as binary 1) or non-seizure epochs (marked as binary 0 ). The vector of classified epochs is then passed through a bitwise logical OR operator, where the number of inputs equals the number of channels being tested. Hence, if an epoch has been detected as a seizure in a single channel, then the final detection decision is that it is a seizure event and the same epoch is marked as a seizure event across all channels. Using this method, the information across all channels is utilized without biasing the algorithm towards their location and montage, and furthermore an electrode disconnection in a single channel would have a minimal effect on the output of the algorithm.

\subsection{Performance metrics}

To assess the utility of each feature for indicating the presence of ictal activity, and the associated computational complexity, five different performance metrics are used here. The sensitivity, specificity and area under the sensitivity-specificity trade-off curve are standard metrics used for assessing the performance of an offline seizure detection algorithm. The relative complexity and overall Figure-Of-Merit are new metrics 
introduced here to allow the computational complexity for online algorithms to be investigated simultaneously.

\subsubsection{Sensitivity}

The sensitivity indicates how many expert marked seizure epochs are correctly identified by the algorithm. A high sensitivity is wanted for good performance. If the number of epochs correctly marked in record $r$ is $T P_{r}$, and the total number of seizure epochs in the record is $S_{r}$, the reported sensitivity is the arithmetic mean of the individual sensitivity values in each record out of a total of $R$ records and is computed as

$$
\text { Sensitivity }=\frac{1}{R} \sum_{r=1}^{R} \frac{T P_{r}}{S_{r}} \times 100 \%
$$

Note that as the sensitivity is calculated on a per-epoch basis this metric does not reflect the number of total seizures that have been detected. For example, a 50\% sensitivity means that 1349 out of 2698 seizure epochs have been correctly detected, but not necessarily that at least one epoch has been detected in every expert marked seizure. To quantify the presence or absence of each seizure, the same sensitivity has been additionally calculated using a windowing method for seizure epochs: when one or more seizure epochs are detected within a single expert marked seizure, then the seizure is considered detected. Hence for each record containing one expert marked seizure, $T P_{r}$ is either zero or one and $S_{r}=1$.It should be noted that there is no windowing for non-seizure epochs and the specificity calculation explained below is valid for both methods of calculating the sensitivity.

\subsubsection{Specificity}

The specificity indicates how many of the expert marked non-seizure epochs are incorrectly marked by the algorithm as seizure activity. A high specificity is wanted for good performance. Specificity is calculated as

$$
\text { Specificity }=\frac{1}{R} \sum_{r=1}^{R} \frac{T N_{r}}{B_{r}} \times 100 \%
$$

where $T N_{r}$ is the number of true negatives (non-seizure epochs correctly classified as nonseizures) and $B_{r}$ is the total number of non-seizure epochs. 


\subsubsection{Sensitivity-specificity trade-off curve and area}

Inevitably there is a trade-off between detection performance of any one feature in terms of the sensitivity and specificity: higher sensitivity can be achieved if lower specificity is tolerated. The normalization and simple detection threshold used in the algorithm (Fig. 1) allows this trade-off to be quantified. When $\beta=1$, the feature $F(e)$ across all epochs will fall below the threshold hence sensitivity will be $0 \%$ and specificity will be $100 \%$. When $\beta$ is set to zero, every epoch will be marked as ictal, and hence sensitivity will be $100 \%$ whilst specificity will be $0 \%$.Varying $\beta$ from zero to one illustrates the trade-off points inbetween. This can be represented as a sensitivity-specificity curve where each point corresponds to the algorithm performance at a different value of $\beta$.

However, due to space limitations it is impractical to plot the full sensitivity-specificity trade-off curve for all 65 features investigated here. Instead the Area Under the Curve (AUC) calculated using trapezoidal estimation is reported. This can be thought of as the summary of the performance of the algorithm across all thresholds [35]: features with higher AUC perform better than features with a lower AUC, and an ideal algorithm would achieve an $\mathrm{AUC}=1$. In addition, each feature has been evaluated to find the lowest threshold at which at least one epoch in every seizure has been detected. At this threshold, the epoch-based sensitivity, specificity and threshold have been reported to reflect how an algorithm would be used in practice where $\beta$ may be selected apriori.

\subsubsection{Relative complexity}

In addition to the above metrics which quantify the detection performance of a feature, for online algorithms it is essential to choose features that not only have high sensitivity and specificity but also have minimal computational complexity. The computational complexity of a feature can be related to the time taken to simulate the feature in MATLAB as features with more processing stages (for example, additions or subtractions) would take longer to calculate in comparison to features with less processing stages. To quantify this, the relative complexity of each feature is calculated. This is found from the simulation time required to generate the feature $F(e)$ and gives a discriminating measure of the computational complexity that is not specific to a particular implementation architecture. As the pre-processing techniques used (time domain, DWT, CWT, FT) will heavily bias the total simulation time, the computational complexity is not compared across the different pre-processing groups, and is instead calculated as a relative measure within each category by dividing the simulation time for the current feature by the minimum simulation time achieved by any feature within the current group. 
The time taken to simulate each feature has been calculated for every $2 \mathrm{~s}$ epoch of EEG data across the entire database (almost 5 million epochs). It has been simulated on a standard desktop computer with a $2.4 \mathrm{GHz}$ processor and $2 \mathrm{~GB}$ RAM running MATLAB version $2010 \mathrm{~b}$. The mode of the relative complexity values is reported here. All times used to calculate the relative complexity have been rounded to the nearest $10 \mu$ s. This precision is determined by repeatedly calculating a single feature within a single $2 \mathrm{~s}$ epoch 100 times and at 5 different times of the day, to ensure the measured time is repeatable.

\subsubsection{Figure-Of-Merit}

For ease of comparison between features, a Figure-Of-Merit (FOM) has been defined as

$$
\mathrm{FOM}=\frac{\mathrm{AUC}}{\text { Relative complexity }}
$$

As the maximum AUC and minimum relative complexity are 1, the FOM is limited to values between $(0,1)$ and higher numbers represent better overall performance.

\subsection{Test database}

Features are compared using adult scalp EEG signals with a total duration of over 172 hours from 24 patients with a total of 47 seizures marked by medical experts for seizure start and end. The data was recorded during routine, ambulatory and long-term monitoring at the National Society of Epilepsy (UK), Katholieke Universiteit Leuven (Belgium) [5, 31] and Freiburg University Hospital (Germany).The database constitutes of 16 channels common to all records and per channel there are $26982 \mathrm{~s}$ seizure epochs and 308630 non-seizure epochs. Non-seizure epochs include background data and may include pre-ictal data, post-ictal data, interictal spikes and/or artefacts, as every epoch within a record that is not classified as a seizure epoch is included in this category. Sections likely to contain pre-ictal and post-ictal discharges or artefacts, have not been removed from the test database in order to test the performance of the features on data similar to what may be expected in a clinical or ambulatory monitoring session. Further details on the test database are provided in the supplementary material.

\section{Results}

The performance of all 65 features implemented and compared here is listed in: Table 2 for features calculated directly from the time domain EEG signal; Table 3 for features 
requiring DWT pre-processing; Table 4 for features with CWT pre-processing; and Table 5 for features with FT based pre-processing. The epoch-based sensitivity and specificity are given for each feature at the threshold value $\beta$. This fixed threshold has been determined by plotting the sensitivity-specificity trade-off curve for every feature (which is not shown here due to space limitations), and then determining the maximum specificity at which at least one epoch in each of the 47 seizures is correctly detected (or in other words, every seizure event has been detected). Consequently, the corresponding epoch-based sensitivity for this specificity is noted. The relative complexity and overall FOM, which do not vary with the threshold $\beta$ and the area under the epoch-based sensitivity-specificity trade-off curve, are also reported. In these tables features are sorted from highest to lowest FOM, and features marked with an asterisk (*) are those which are expected to decrease during a seizure and so have been calculated using $\mathrm{N}(\mathrm{e})<\beta$ in Fig. 1. Some specific comments on the results in each feature group are given below before conclusions are drawn in Section 4.

\subsection{Time domain features}

From Table 2, the largest area under the sensitivity-specificity trade-off curve is achieved by the line length of the EEG signal, and closely followed by nonlinear energy, variance, energy and maximum. The lowest relative complexity is achieved by line length, energy, maximum, minimum, total maximum and minimum, and mean. All six features were simulated in $10 \mu \mathrm{s}$, the resolution of the simulation time in the study, and hence have been taken as the baseline for relative complexity.

Overall, the line length has the largest Figure-Of-Merit as it has both maximum AUC and minimum relative complexity. The worst performer is Shannon entropy as it has the highest relative complexity, although other features have lower AUC. However, while the difference between maximum AUC (0.77) and minimum AUC (0.53) is noticeable, the spread of the relative complexity is largely the deciding factor for the FOM in Table 2.

\subsection{DWT-based features}

Table 3 shows the performance of the eight DWT features evaluated in each of the four frequency ranges (D3: 12.5-25 Hz; D4: 6.25-12.5 Hz; D5: 3.125-6.25 Hz; A5: 0.16$3.125 \mathrm{~Hz}$ ). Looking at only the AUC value it is clear that the relative power in the D3, D4 and D5 frequency ranges gives the best detection performance. Having an AUC>0.8, these features have noticeably better seizure detection accuracy than all of the other features studied in this paper. Relative power in the D3 and D5 frequency bands perform 
equally the best with $\mathrm{AUC}=0.83$. It can also be seen that within each feature the highest performance is consistently obtained by considering D5 $(3.125-6.25 \mathrm{~Hz})$ frequencies. When the relative complexity is analysed separately a clear divide between the different frequency ranges can be seen. Features in the D3 frequency range have the lowest relative complexity. This is to be expected, as the DWT is a multi-scale analysis and so components in the D3 $12.5-25 \mathrm{~Hz}$ band must be calculated prior to further decomposition in to the D4, D5 and A5 frequency bands and then the processing to generate the other features. Similarly, it is clear that features calculated in the D4 frequency range have lower relative complexity than those requiring a further level of decomposition to D5 and A5. Within features evaluated for the same frequency range, such as D3, it can be seen that the raw coefficients have the lowest relative complexity, followed by energy, variances and entropy. Relative Scale Energy (RSE) is a special case in terms of relative complexity, as the calculation of RSE in any frequency range requires energy in all the frequency bands to be computed. Hence the relative complexity of RSE in any frequency range is similar to features in the maximum decomposition stage, D5 and A5 frequencies. Across all DWT-based features, the relative power in the D3 frequency range has the highest FOM. There is $12.5 \%$ drop in FOM between this feature and the runners-up: energy (D3) and entropy (D3).

\subsection{CWT-based features}

Table 4 shows that the highest AUC is achieved by energy and standard deviation of the energy. Energy also has the lowest relative complexity and hence the highest FOM.

\subsection{FT-based features}

Table 5 shows that spectral entropy (in D3 and D5), power (in D3 and D5) and total spectral power, all perform well. Looking at relative complexity, all features except spectral edge frequency perform identically - any difference has been limited by the precision of relative complexity calculation. Consequently the spectral entropy (D3 and D5) and power (D5) have the highest Figure-Of-Merit. Total spectral power and power (D3) also perform well, with less than 3\% difference in Figure-Of-Merit from the best performer. It should be noted that unlike the DWT-based features, with the FT there is no

difference in the simulation time across different frequency ranges because a 512-point Fast Fourier Transform is calculated giving information in all frequency ranges at the same time. 


\subsection{Overall performance}

To illustrate the full performance trade-off, the epoch-based sensitivity and specificity is plotted in Fig. 2 for the highest performing feature in terms of FOM, from each of the pre-processing categories. The presence or absence of each seizure event as a fraction of 47 expert marked seizures is also plotted for the same specificity and threshold $\beta$. Both sensitivity metrics demonstrate that the DWT relative power in the D3 $12.5-25 \mathrm{~Hz}$ range achieves the best seizure detection performance across all features. Here, $80 \%$ epochbased sensitivity is achieved for $70 \%$ specificity whilst detecting a section of every seizure (100\% seizures detected). For the other features, $80 \%$ epoch-based sensitivity is achieved at $60 \%$ specificity for line length, $40 \%$ specificity for CWT energy and $50 \%$ specificity for FT-based spectral entropy in the D3 range.

\section{Discussion}

When only the area under the sensitivity-specificity trade-off curve is used to compare between features considered here, relative power in the $12.5-25 \mathrm{~Hz}$ and $3.125-6.25 \mathrm{~Hz}$ frequency ranges performed the best in terms of seizure detection performance, while relative power in the $6.25-12.5 \mathrm{~Hz}$ frequency range and line length ranked in the top four respectively. This is a surprising result since only two of the 97 considered publications from 2000-2010 [19, 20] have used relative power and a further two publications [17, 22] have used line length to extract epileptic seizures from adult scalp EEG. This can be compared to the number of publications using variance/standard deviation (13) and Shannon entropy (5); DWT energy (5) and variance (5); and FT spectral entropy (7) as given in Table 1.

When computational complexity is also considered, the DWT relative power in the 12.5$25 \mathrm{~Hz}$ frequency range gives the best Figure-Of-Merit (0.80). Thus, provided the DWT can be implemented within the power constraints of an online EEG system [15, 23], this feature is the best choice on both fronts. Although relative computational complexity has not been compared between different feature groups in order to avoid bias due to the implementation of the pre-processing method, it should be noted that the computational complexity of implementing a time domain feature with low relative complexity, will nevertheless be lower than features from other feature groups, as time domain features do not require an extra pre-processing stage. Given this, the line length also has a very high FOM (0.77). Comparing the best CWT and FT features to the line length, the best features in these feature categories have both a lower AUC than line length and require an additional pre-processing stage, and would thus not be an optimal choice. Consequently 
the best performing features are line length and DWT relative power in $12.5-25 \mathrm{~Hz}$ frequency band, and the choice of which feature(s) should be selected will depend on the performance and power consumption requirements of the seizure detection system. Regardless, it is interesting to note that line length has been previously reported to be amongst the best performing features for neonatal seizures [10]. (The other features in [10] performed differently in this study although this performance variation is expected, due to the well known differences between adult and neonatal EEG [6].) Considering only adult, scalp EEG based seizure detection - as the most common type of EEG recording, and also the EEG recording that is most frequently contaminated with biological artefacts (such as eye blinks and muscle activity) making the accurate detection of seizures more difficult - the most comprehensive feature comparison study compares 16 features [30] whilst two other studies compare 9 features $[19,20]$. In [20], relative power is reported to be one of the best features for seizure detection, but the study did not discuss performance variation over frequency. Although separate publications have reported line length and relative power to be amongst the best features, it is important to note that this study not only evaluates the largest number of features, but also addresses performance variation across frequencies, on minimally pre-processed scalp EEG of adult patients and also takes into consideration the computational complexity of each feature, thus evaluating performance as a trade-off between detection accuracy (AUC) and computational complexity.

In conclusion, this study quantifies the performance of 65 features tested on minimally pre-processed human scalp EEG, in terms of their sensitivity, specificity, area under the sensitivity-specificity trade-off curve and relative computational complexity. Here, DWT relative power evaluated in the $12.5-25 \mathrm{~Hz}$ frequency range and line length calculated on the raw EEG signal were found to be the best performers, although, these features have only been used in 4 seizure detection algorithms published in the last decade. This work thus provides key new insights into seizure detection algorithm design and in particular, these results allow designers of emerging online seizure detection algorithms to better focus their design effort.

\section{Acknowledgements}

The research leading to these results has received funding from the European Research Council under the European Community's 7th Framework Programme (FP7/20072013)/ERC grant agreement no.239749. 


\section{References}

1. Agostinho PR, Haddad S, De Lima JA, Serdijn WA, Saotome O (2008) An ultra low power CMOS pA/V transconductor and its application to wavelet filters. Analog Integrated Circuits and Signal Processing 57:19-27

2. Badawy RAB, Pillay N, Jettec N, Wiebec S, Federico P (2011) A blinded comparison of continuous versus sampled review of video-EEG monitoring data. Clin Neurophysiol 122:1086-1090

3. Casson AJ, Yates DC, Smith SJ, Duncan JS, Rodriguez-Villegas E (2010) Wearable electroencephalography. IEEE EMBS Mag 29:44-56

4. Casson AJ, Rodriguez-Villegas E (2011) A 60 pW gmC Continuous Wavelet Transform Circuit for Portable EEG Systems. J Solid-State Circuits 46:1406-1415

5. DeClercq W, Vergult A, Vanrumste B, Van Paesschen W, Van Huffel S (2006) Canonical correlation analysis applied to remove muscle artifacts from the electroencephalogram. IEEE Trans Biomed Eng 53:2583-2587

6. Faul S, Temko A, Marnane W (2009) Age-independant seizure detection. In: Proceedings of the 31st international conference of IEEE Engineering Medicine Biology Society, Minnesota, IEEE, Piscataway, pp 6612-6615

7. Goncharova II, McFarland DJ, Vaughan TM, Wolpaw JR (2003) EMG contamination of EEG: spectral and topographical characteristics. Clin Neurophysiol 114:1580-1593

8. Gotman J, Ives JR, Gloor P (1981) Frequency content of EEG and EMG at seizure onset: possibility of removal of EMG artefact by digital filtering. Electroenceph Clin Neurophysiol 52:626-639

9. Gotman J (1999) Automatic detection of seizures and spikes. J Clin Neurophysiol $16: 130-140$

10. Greene BR, Faul S, Marnane WP, Lightbody G, Korotchikova I, Boylan GB (2008) A comparison of quantitative EEG features for neonatal seizure detection. Clin Neurophysiol 119:1248-1261

11. Guerrero-Mosquera C, Trigueros AM, Fraco JI, Navia-Vàzquez (2010) New feature extraction approach for epileptic EEG signal detection using time-frequency distributions. Med Biol Eng Comput. 48: 321-333

12. Hall Jr CW, Sarkar A (2011) Mutual information in natural position order of electroencephalogram is significantly increased at seizure onset. Med Biol Eng Comput. 49:133-141

13. Harreby KR, Sevcencu C, Struijik JJ (2011) Early seizure detection in rats based on vagus nerve activity. Med Biol Eng Comput. 49:143-151 
14. Hongmin L, Yigang H, Sun Y (2008) Detection of Cardiac Signal Characteristic Point Using Log-Domain Wavelet Transform Circuits. Circuits Syst Signal Process 27:683-698

15. Kamboh AM, Raetz M, Oweiss KG, Mason A (2007) Area-Power Efficient VLSI Implementation of Multichannel DWT for Data Compression in Implantable Neuroprosthetics. IEEE Trans Biomed Circuits Systems 1:128-135

16. Kelly KM, Shiaud DS, Kernd RT, Chiend JH, Yang MCK, Yandora KA, Valeriano JP, Halford JJ, Sackellares JC (2010) Assessment of a scalp EEG-based automated seizure detection system. Clin Neurphysiol 121:1832-1843

17. Kim H, Rosen J (2010) Epileptic Seizure Detection -An AR Model Based Algorithm for Implantable Device. In: Proceedings of the 32nd international conference of IEEE Engineering Medicine Biology Society, Buenos Aires, IEEE, Piscataway, pp 5541

18. Koubeissi MZ, Syed TU (2011) Inpatient video-EEG monitoring: How much shall we review? Clin Neurophysiol 122:1065-1066

19. Kuhlmann L, Cook MJ, Fuller K, Grayden DB, Burkitt AN, Mareels IMY (2008) Correlation analysis of seizure detection features. In: Proceedings of the international conference on Intelligent Sensors, Sensor Networks and Information Processing, Sydney, IEEE, Piscataway, pp 309-314

20. Kuhlmann L, Burkitt AN, Cook MJ, Fuller K, Grayden DB, Seiderer L, Mareels IMY (2009). Seizure Detection Using Seizure Probability Estimation: Comparison of Features Used to Detect Seizures. Ann Biomed Eng 37:2129-2145

21. Lesser RP (2009) Epilepsy: Does continuous EEG monitoring improve seizure control? Nat Rev Neurol 5:581-582

22. McEvoy RP, Faul S, Marnane WP (2010) Ambulatory REACT: Real-time seizure detection with a DSP microprocessor. In: Proceedings of the international conference of IEEE Engineering Medicine Biology Society, Buenos Aires, IEEE, Piscataway, pp 2443-244

23. Narasimhan S, Chiel HJ, Bhunia S (2010) Ultra-Low-Power and Robust DigitalSignal-Processing Hardware for Implantable Neural Interface Microsystems. IEEE Trans Biomed Circuits Systems 4:1-10

24. Nuwer MR, Comi G, Emerson R, Fuglsang-Frederiksen A, Guerit J, Hinrichs H, Ikeda A, Luccas FJC, Rappelsburger P (1998) IFCN standards for digital recording of clinical EEG. Electroenceph Clin Neurophysiol 106:259-261

25. O’Donnell RD, Berkhout J, Adey WR (1974) Contamination of scalp EEG spectrum during contraction of cranio-facial muscles. Electroencephalogr Clin Neurophysiol $37: 145-151$ 
26. Raghunathan S, Gupta SK, Markandeya HS, Roya K, Irazoqui PP (2010) A hardware-algorithm co-design approach to optimize seizure detection algorithms for implantable applications. J Neuroscience Methods 1:106-117

27. Shiau D, Halford JJ, Kelly KM, Kern RT, Inman M, Chien J, Pardalos PM, Yang MCK, Sackellares JC (2010) Signal regularity-based automated seizure detection system for scalp EEG monitoring. Cybern Syst Anal 46:922-935

28. Sisodiya S (2007) Etiology and management of refractory epilepsies. Nat Clin Pract Neuro 3:320-330

29. Tito M, Cabrerizo M, Ayala M, Jayakar P, Adjouadi M(2009) Seizure Detection: An Assessment of Time-and Frequency-Based Features in a Unified Two-Dimensional Decisional Space Using Nonlinear Decision Functions. J Clin Neurophysiol 26:381391

30. van Putten MJAM, Kind T, Visser F, Lagerburg V (2005) Detecting temporal lobe seizures from scalp EEG recordings: A comparison of various features. Clin Neurophysiol 116:2480-2489

31. Vergult A, De Clercq Q, Palmini A, Vanrumste B, Dupont P, Van Huffel S, Van Paesschen W (2007) Improving the Interpretation of Ictal Scalp EEG: BSS-CCA Algorithm for Muscle Artifact Removal. Epilepsia 48:950-958

32. Verma N, Shoeb A, Bohorquez J, Dawson J, Guttag J, Chandrakasan AP (2010) A Micro-Power EEG Acquisition SoC With Integrated Feature Extraction Processor for a Chronic Seizure Detection System. J Solid-State Circuits 45:804-816

33. Wang A, Chandrakasan AP. A 180-mV Subthreshold FFT Processor Using a Minimum Energy Design Methodology. IEEE J Solid-State Circuits 40:310-319

34. Zandi AS, Javidan M, Dumont GA, Tafreshi R (2010) Automated real-time epileptic seizure detection in scalp EEG recordings using an algorithm based on wavelet packet transform. IEEE Trans Biomed Eng 57:1639-1651

35. Zweig MH, Campbell G (1993) Receiver-operating characteristic (ROC) plots: a fundamental evaluation tool in clinical medicine. Clin Chem 39:561-577 
Table 1 The sixty-five features compared in this study, including features calculated in frequency bands D3, D4, D5 and A5 marked with $\dagger$

\begin{tabular}{|c|c|c|}
\hline Category & Feature & Number of papers \\
\hline \multirow[t]{17}{*}{ Time domain } & Complexity & 4 \\
\hline & Energy/power & 4 \\
\hline & Fractal dimension & 3 \\
\hline & Kurtosis & 3 \\
\hline & Line length & 2 \\
\hline & Maximum & 2 \\
\hline & Mean & 2 \\
\hline & Minimum & 2 \\
\hline & Mobility & 3 \\
\hline & Non-linear energy & 2 \\
\hline & Relative derivative & 2 \\
\hline & Shannon entropy & 5 \\
\hline & Skewness & 2 \\
\hline & Total maxima and minima & 2 \\
\hline & Variance/standard deviation & 13 \\
\hline & Zero crossing & 4 \\
\hline & Zero crossing of first derivative & 2 \\
\hline \multirow[t]{8}{*}{ DWT } & Bounded variation $\dagger$ & 2 \\
\hline & Coefficients $\dagger$ & 4 \\
\hline & Energy $\dagger$ & 5 \\
\hline & Entropy $\dagger$ & 4 \\
\hline & Relative bounded variation $\dagger$ & 2 \\
\hline & Relative power $\dagger$ & 2 \\
\hline & Relative scale energy $\dagger$ & 2 \\
\hline & Variance/standard deviation $\dagger$ & 5 \\
\hline \multirow[t]{4}{*}{ CWT } & Coefficient z-score & 1 \\
\hline & Energy & 2 \\
\hline & Entropy & 1 \\
\hline & Standard deviation of energy & 1 \\
\hline \multirow[t]{6}{*}{ FT } & Median frequency & 2 \\
\hline & Peak frequency & 3 \\
\hline & Power $\dagger$ & 4 \\
\hline & Spectral edge frequency & 2 \\
\hline & Spectral entropy $\dagger$ & 7 \\
\hline & Total spectral power & 3 \\
\hline
\end{tabular}


Table 2 Performance of time domain features. Features denoted with * decrease during seizure.

\begin{tabular}{|c|c|c|c|c|c|c|}
\hline \multirow[t]{2}{*}{ Feature } & $\begin{array}{l}\text { Sensitivity } \\
(\%)\end{array}$ & $\begin{array}{l}\text { Specificity } \\
(\%)\end{array}$ & $\beta$ & \multirow[t]{2}{*}{ AUC } & \multirow[t]{2}{*}{$\begin{array}{l}\text { Relative } \\
\text { complexity }\end{array}$} & \multirow[t]{2}{*}{ FOM } \\
\hline & \multicolumn{3}{|c|}{$\begin{array}{l}\text { For } \geq 1 \text { epoch detected in every } \\
\text { seizure }\end{array}$} & & & \\
\hline Line length & 85.54 & 52.06 & 0.40 & 0.77 & 1.0 & 0.77 \\
\hline Energy/power & 95.95 & 15.59 & 0.02 & 0.74 & 1.0 & 0.74 \\
\hline Maximum & 88.82 & 35.46 & 0.25 & 0.74 & 1.0 & 0.74 \\
\hline Minimum & 89.01 & 25.71 & 0.20 & 0.72 & 1.0 & 0.72 \\
\hline Total minima and maxima & 98.10 & 00.30 & 0.55 & 0.67 & 1.0 & 0.67 \\
\hline Mean & 78.59 & 35.45 & 0.10 & 0.64 & 1.0 & 0.64 \\
\hline Non-linear energy & 84.39 & 51.55 & 0.15 & 0.76 & 2.0 & 0.38 \\
\hline Fractal dimension & 72.08 & 25.18 & 0.80 & 0.53 & 4.0 & 0.13 \\
\hline Zero crossing & 64.63 & 47.62 & 0.75 & 0.61 & 5.0 & 0.12 \\
\hline $\begin{array}{l}\text { Zero crossing of first } \\
\text { derivative }\end{array}$ & 72.07 & 33.00 & 0.92 & 0.60 & 5.0 & 0.12 \\
\hline Variance & 95.98 & 15.67 & 0.02 & 0.75 & 7.0 & 0.11 \\
\hline Relative derivative & 86.28 & 19.47 & 0.10 & 0.66 & 12.0 & 0.05 \\
\hline Mobility & 58.94 & 60.30 & 0.75 & 0.63 & 14.0 & 0.05 \\
\hline Skewness & 89.53 & 11.19 & 0.10 & 0.58 & 14.0 & 0.04 \\
\hline Kurtosis & 78.56 & 18.85 & 0.02 & 0.54 & 14.0 & 0.04 \\
\hline Complexity & 80.13 & 27.01 & 0.30 & 0.64 & 20.0 & 0.03 \\
\hline Shannon entropy $*$ & 92.95 & 12.49 & 0.75 & 0.63 & 31.0 & 0.02 \\
\hline
\end{tabular}


Table 3 Performance of DWT-based features. Features denoted with * decrease during seizure.

\begin{tabular}{|c|c|c|c|c|c|c|c|}
\hline \multirow[t]{2}{*}{ Feature } & \multirow{2}{*}{$\begin{array}{l}\text { Frequ } \\
\text {-ency } \\
\text { range }\end{array}$} & $\begin{array}{l}\text { Sensitivity } \\
(\%)\end{array}$ & $\begin{array}{l}\text { Specificity } \\
(\%)\end{array}$ & $\beta$ & \multirow[t]{2}{*}{ AUC } & \multirow{2}{*}{$\begin{array}{l}\text { Rela } \\
\text {-tive } \\
\text { compl } \\
\text {-exity }\end{array}$} & \multirow[t]{2}{*}{ FOM } \\
\hline & & \multicolumn{3}{|c|}{$\begin{array}{l}\text { For } \geq 1 \text { epoch detected in every } \\
\text { seizure }\end{array}$} & & & \\
\hline Relative power & D3 & 71.32 & 79.67 & 0.02 & 0.83 & 1.04 & 0.80 \\
\hline Energy & D3 & 88.02 & 23.20 & 0.08 & 0.71 & 1.01 & 0.70 \\
\hline Entropy & D3 & 87.47 & 25.52 & 0.06 & 0.71 & 1.01 & 0.70 \\
\hline Coefficients & D3 & 56.43 & 74.83 & 0.45 & 0.69 & 1.00 & 0.69 \\
\hline Variance & D3 & 88.00 & 23.14 & 0.08 & 0.71 & 1.04 & 0.69 \\
\hline Relative power & D4 & 73.28 & 76.12 & 0.02 & 0.81 & 1.35 & 0.60 \\
\hline Energy & D4 & 93.18 & 13.82 & 0.04 & 0.70 & 1.34 & 0.53 \\
\hline Entropy & D4 & 90.33 & 18.40 & 0.04 & 0.70 & 1.34 & 0.53 \\
\hline Variance & D4 & 90.33 & 18.62 & 0.06 & 0.70 & 1.34 & 0.52 \\
\hline Bounded variation $*$ & D3 & 61.26 & 43.80 & 0.45 & 0.53 & 1.02 & 0.52 \\
\hline Relative bounded variation & D3 & 38.98 & 71.39 & 0.86 & 0.54 & 1.03 & 0.52 \\
\hline Coefficients & D4 & 48.63 & 76.71 & 0.50 & 0.66 & 1.33 & 0.49 \\
\hline Relative power & D5 & 62.99 & 84.67 & 0.04 & 0.83 & 1.68 & 0.49 \\
\hline Relative bounded variation & D4 & 49.52 & 68.40 & 0.84 & 0.63 & 1.36 & 0.46 \\
\hline Bounded variation & D4 & 42.26 & 74.01 & 0.88 & 0.61 & 1.35 & 0.46 \\
\hline Energy & D5 & 86.54 & 33.32 & 0.08 & 0.75 & 1.66 & 0.45 \\
\hline Variance & D5 & 86.44 & 33.54 & 0.08 & 0.75 & 1.66 & 0.45 \\
\hline Entropy & D5 & 86.96 & 32.95 & 0.06 & 0.75 & 1.66 & 0.45 \\
\hline Variance & A5 & 96.23 & 13.68 & 0.01 & 0.73 & 1.66 & 0.44 \\
\hline Entropy & A5 & 95.11 & 16.83 & 0.01 & 0.73 & 1.66 & 0.44 \\
\hline Energy & A5 & 96.40 & 12.75 & 0.01 & 0.73 & 1.66 & 0.44 \\
\hline Relative power & A5 & 80.94 & 51.28 & 0.01 & 0.73 & 1.68 & 0.44 \\
\hline Coefficients & A5 & 39.34 & 84.90 & 0.50 & 0.68 & 1.65 & 0.41 \\
\hline Bounded variation & A5 & 39.57 & 83.38 & 0.88 & 0.67 & 1.68 & 0.40 \\
\hline Relative bounded variation & A5 & 56.68 & 69.77 & 0.78 & 0.67 & 1.69 & 0.40 \\
\hline Coefficients & D5 & 37.01 & 84.64 & 0.60 & 0.65 & 1.65 & 0.39 \\
\hline Relative bounded variation & D5 & 42.01 & 79.98 & 0.88 & 0.66 & 1.69 & 0.39 \\
\hline Bounded variation & D5 & 30.33 & 87.67 & 0.94 & 0.66 & 1.68 & 0.39 \\
\hline Relative scale energy & D5 & 50.95 & 72.36 & 0.60 & 0.65 & 1.66 & 0.39 \\
\hline Relative scale energy & D3 & 76.91 & 34.38 & 0.15 & 0.62 & 1.66 & 0.37 \\
\hline Relative scale energy & D4 & 62.41 & 51.97 & 0.35 & 0.61 & 1.66 & 0.37 \\
\hline Relative scale energy $*$ & A5 & 81.44 & 25.88 & 0.80 & 0.57 & 1.67 & 0.34 \\
\hline
\end{tabular}


Table 4 Performance of CWT-based features.

\begin{tabular}{|c|c|c|c|c|c|c|}
\hline \multirow[t]{2}{*}{ Feature } & $\begin{array}{l}\text { Sensitivity } \\
(\%)\end{array}$ & $\begin{array}{l}\text { Specificity } \\
(\%)\end{array}$ & $\beta$ & \multirow[t]{2}{*}{ AUC } & \multirow[t]{2}{*}{$\begin{array}{l}\text { Relative } \\
\text { complexity }\end{array}$} & \multirow[t]{2}{*}{ FOM } \\
\hline & \multicolumn{3}{|c|}{$\begin{array}{l}\text { For } \geq 1 \text { epoch detected in every } \\
\text { seizure }\end{array}$} & & & \\
\hline Energy & 81.74 & 37.61 & 0.10 & 0.72 & 1.00 & 0.72 \\
\hline $\begin{array}{l}\text { Standard deviation of } \\
\text { energy }\end{array}$ & 83.94 & 30.12 & 0.06 & 0.70 & 1.03 & 0.68 \\
\hline Coefficient z-score & 74.62 & 50.90 & 0.20 & 0.69 & 1.06 & 0.65 \\
\hline Entropy & 48.36 & 71.09 & 0.90 & 0.63 & 1.56 & 0.40 \\
\hline
\end{tabular}


Table 5 Performance of FT-based features.

\begin{tabular}{|l|l|l|l|l|l|l|l|l|}
\hline Feature & Frequ & $\begin{array}{l}\text { Sensitivity } \\
(\%)\end{array}$ & $\begin{array}{l}\text { Specificity } \\
(\%)\end{array}$ & $\beta$ & AUC & Rela & FOM \\
\cline { 3 - 8 } & range & \multicolumn{2}{l}{$\begin{array}{l}\text { For } \geq 1 \text { epoch detected in } \\
\text { every seizure }\end{array}$} & & $\begin{array}{l}\text {-tive } \\
\text { compl- } \\
\text { exity }\end{array}$ & \\
\hline Spectral entropy & D3 & 87.98 & 33.65 & 0.02 & 0.74 & 1.0 & 0.74 \\
\hline Spectral entropy & D5 & 81.68 & 40.11 & 0.06 & 0.73 & 1.0 & 0.73 \\
\hline Power & D5 & 85.07 & 33.29 & 0.08 & 0.73 & 1.0 & 0.73 \\
\hline Total spectral power & - & 87.66 & 24.74 & 0.06 & 0.72 & 1.0 & 0.72 \\
\hline Power & D3 & 89.66 & 21.04 & 0.06 & 0.72 & 1.0 & 0.72 \\
\hline Spectral entropy & A5 & 91.63 & 22.22 & 0.01 & 0.70 & 1.0 & 0.70 \\
\hline Power & A5 & 90.73 & 22.90 & 0.02 & 0.70 & 1.0 & 0.70 \\
\hline Spectral entropy & - & 83.45 & 32.17 & 0.10 & 0.64 & 1.0 & 0.64 \\
\hline Power & D4 & 92.45 & 19.38 & 0.02 & 0.69 & 1.0 & 0.69 \\
\hline Peak frequency & D4 & 90.42 & 19.32 & 0.06 & 0.68 & 1.0 & 0.68 \\
\hline Median frequency & - & 92.23 & 17.01 & 0.10 & 0.64 & 1.0 & 0.64 \\
\hline Spectral edge frequency & - & 56.24 & 49.37 & 0.80 & 0.55 & 7.8 & 0.07 \\
\hline
\end{tabular}




\section{Figure legends}

Fig. 1 Flowchart of the single channel seizure detection algorithm used to compare features.

Fig. 2 Sensitivity-specificity trade-off curves as the detection threshold $\beta$ is varied. Curves are plotted for the best performing feature (in terms of FOM) from each of the feature groups (time domain, DWT, CWT, FT) 


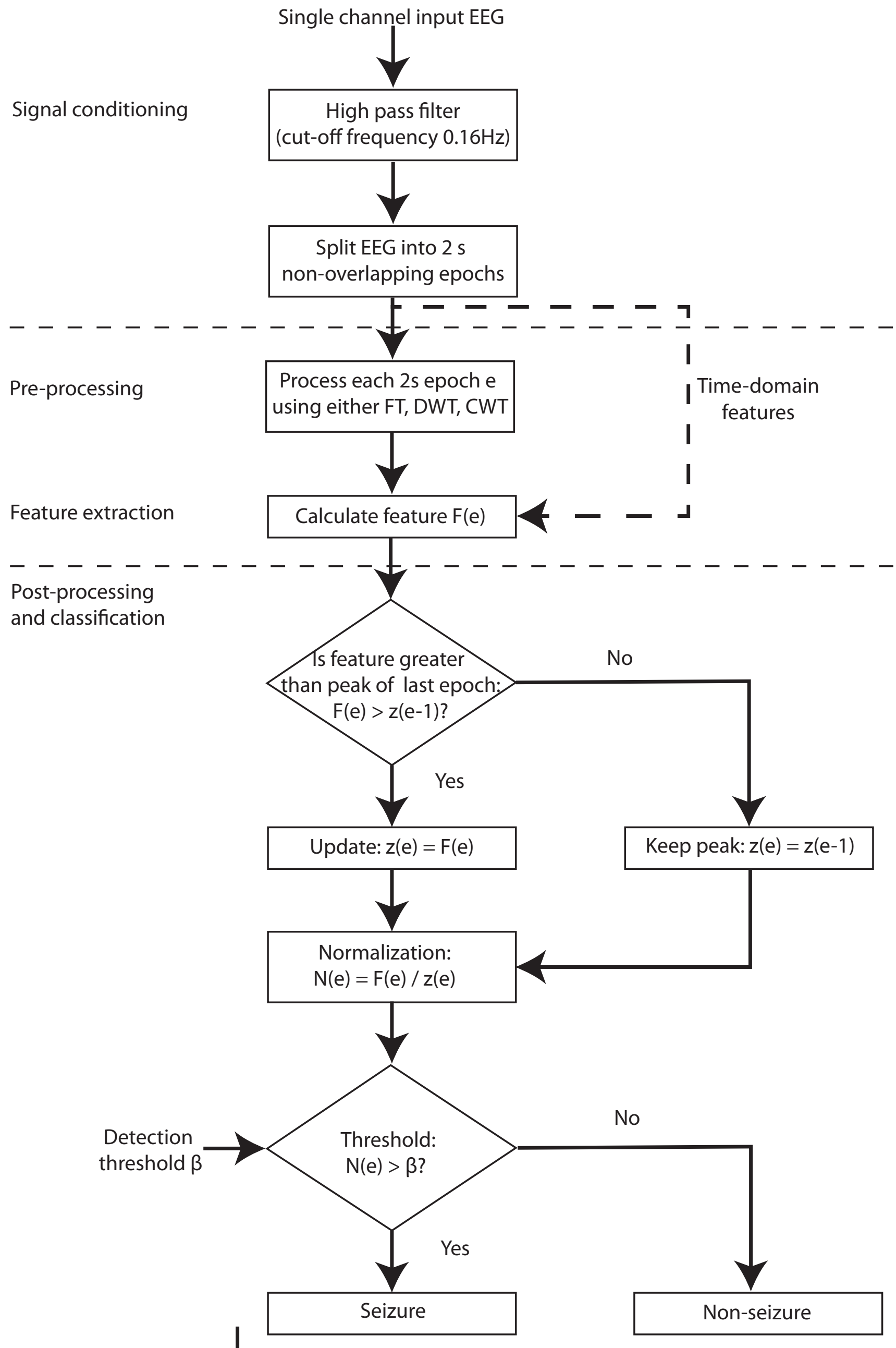




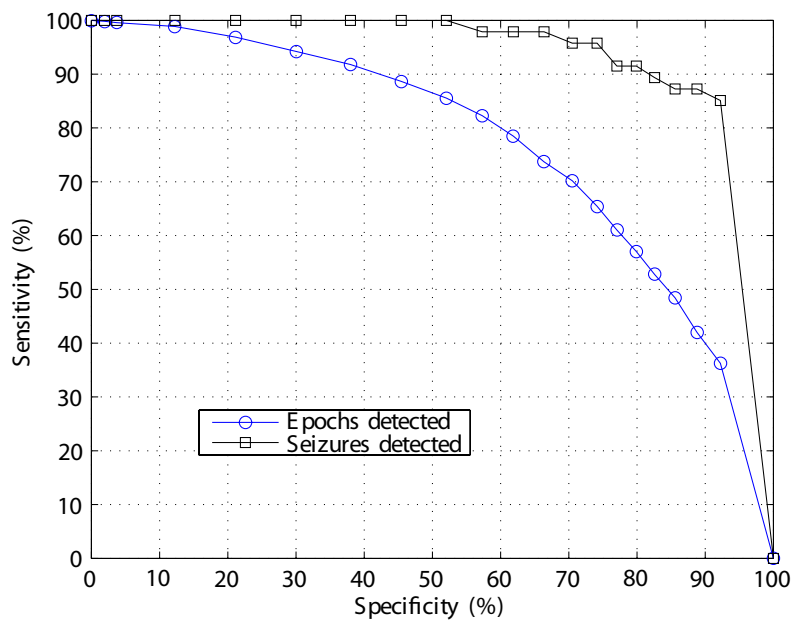

(a) Time domain feature: Line length.

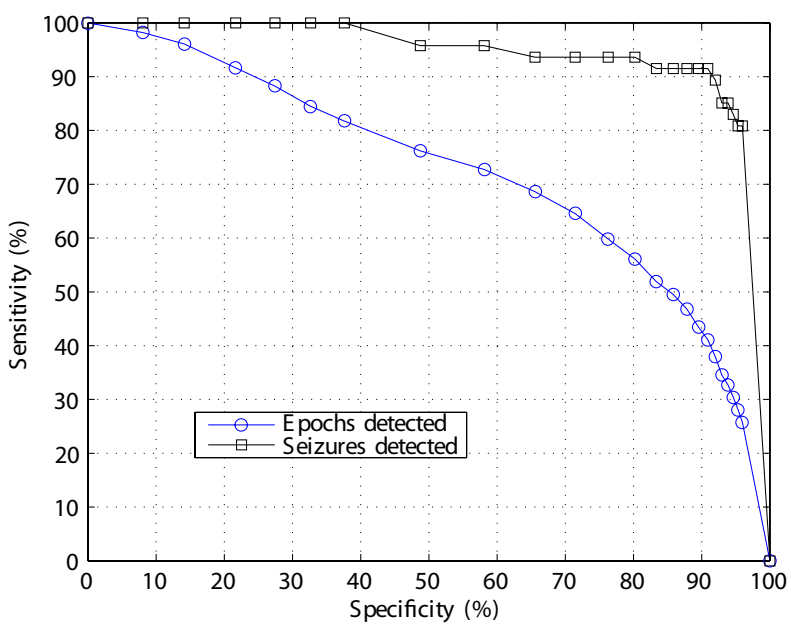

(c) CWT-based feature: Energy

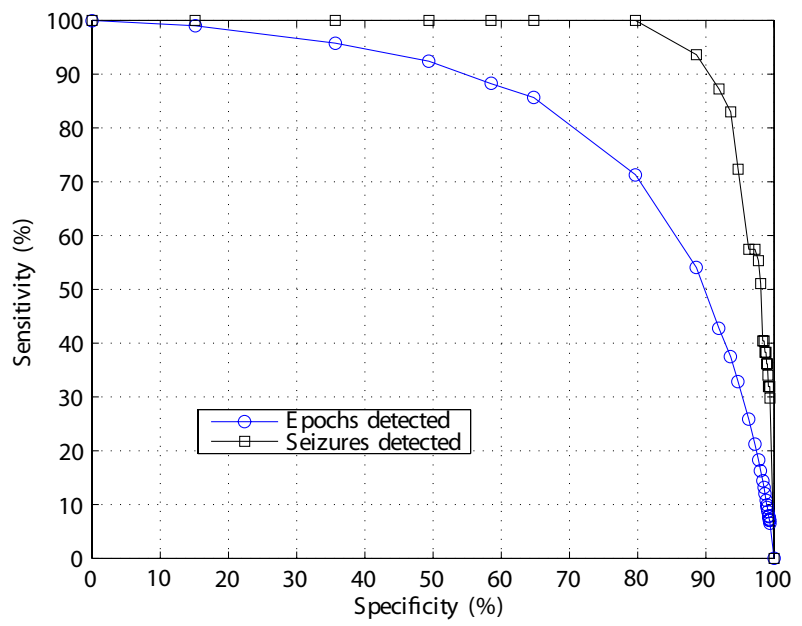

(b) DWT-based feature: Relative power in D3 $12.5-25 \mathrm{~Hz}$.

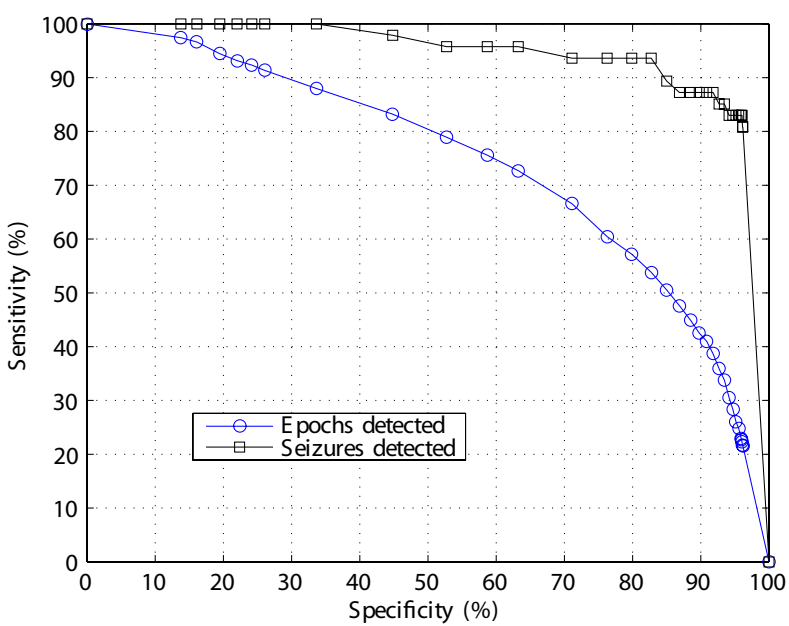

(d) FT-based feature: Spectral entropy at 12.5-25 Hz. 


\section{Supplementary material}

This supplement is divided in to three parts: literature review details are given in Section 1; full calculations of the features implemented in this study is described in Section 2; and details on the test EEG database are covered in Section 3.

\section{Literature review details}

This section is further divided into three parts. Section 1.1 discusses the inclusion criteria used to select seizure detection publications for this literature review and full details on the 97 short-listed publications have also been covered here. Next, the method used to segment the algorithms described in the 97 publications into pre-processing, feature extraction and post-processing methods is described in Section 1.2. It is important to note that any processing carried out before or after feature extraction will affect the performance of the feature. Hence, four popular pre-processing techniques have been selected in Section 1.3 and the study has been limited to features that are calculated after these four processing methods. Full calculations of the features implemented in the study are given in Section 2.

Post-processing and classification after feature extraction is kept constant for all features in this study and described together with the seizure detection algorithm in the main text.

\subsection{Publications reviewed}

Ninety-seven publications dealing with seizure detection algorithms using scalp EEG data from adult patients were used to select the features to investigate in this study. These publications were short-listed by searching IEEExplore and Google Scholar for articles published between 2000 and 2010 which contain the following words in the title: 'ictal', 'seizure' or 'EEG' and did not contain the terms: 'neonatal', 'child', 'animal', 'onset' or 'predict'. Returned articles were reviewed to ensure that only seizure detection algorithms tested on scalp EEG recordings, and not tested solely on neonatal or paediatric patients, were included. It should be noted that many publications do not specify the age group of the patients, and such publications have been included here if their test EEG database contained scalp EEG records. There are 45 journal publications and 52 conference publications. Full details of the 97 publications are given below. 


\section{References}

1. Abibullaev B, Kim MS, Seo HD (2010) Seizure Detection in Temporal Lobe Epileptic EEGs Using the Best Basis Wavelet Functions. J Medical Systems 34:755765

2. Acar E, Bingol CA, Bingol H, Bro R, Yener B (2007) Seizure Recognition on Epilepsy Feature Tensor. In: Proceedings of the 29th international conference of the IEEE Engineering Medicine Biology Society, Lyon, IEEE, Piscataway, pp 4273 4276

3. Adeli H, Ghosh-Dastidar S, Dadmehr N (2007) A wavelet-chaos methodology for analysis of EEGs and EEG subbands to detect seizure and epilepsy. IEEE Trans Biomed Eng 54:205-211

4. Alipoor M, Pooyan M, Suratgar AA (2010) Classification of EEG Signals in Four Groups, Including Healthy Subjects with Open/Closed Eyes and Epilepsy Subjects with/without Seizure by PSD Estimate (Using the Multitaper Method) and ANN. In: Proceedings of the 5th international symposium on Health Informatics and Bioinformatics, Antalya, IEEE, Piscataway, pp 98-103

5. Alkan A, Kiymik MK (2006) Comparison of AR and Welch methods in epileptic seizure detection. J Medical Systems 30:413-419

6. Alkan A, Koklukaya E, Subasi A (2005) Automatic seizure detection in EEG using logistic regression and artificial neural network. J Neuroscience Methods 148:167176

7. Almeida P, Gomes P, Sales F, Nogueira A, Dourado A (2010). Ontology and Knowledge Management System on Epilepsy and Epileptic Seizures. In:

Proceedings of the 3rd international workshop on semantic web applications and tools for the life sciences, Berlin

8. Aydin S, Saraoglu HM, Kara S (2009) Log Energy Entropy-Based EEG Classification with Multilayer Neural Networks in Seizure. Ann Biomed Eng $37: 2626-2630$

9. Battiston JJ, Darcey TM, Siegel AM, Williamson PD, Barkan HI, Akay M, Thadani VM, Roberts DW (2003) Statistical Mapping of Scalp-Recorded Ictal EEG Records Using Wavelet Analysis. Epilepsia 44:664-672

10. Bedeeuzzaman VM, Farooq O, Khan YU (2010) Automatic Seizure Detection Using Higher Order Moments. In: Proceedings of the international conference on Recent Trend in Information, Telecommunication and Computing, Kerala, IEEE CPS, California, pp 159-163

11. Bianchi AM, Panzica F, Tinello F, Franceschetti S, Cerutti S, Baselli G (2003) Analysis of multichannel EEG synchronization before and during generalized 
epileptic seizures. In: Proceedings of the 1st international IEEE EMBS Conference on Neural Engineering, Capri Island, IEEE, Piscataway, pp 39-42

12. Berdakh A, Seo Hee D (2009) Epileptic Seizures Detection Using Continuous Time Wavelet Based Artificial Neural Networks. In: Sixth international conference on Information Technology: New Generations, Las Vegas, IEEE CPS, California, pp 1456-1461

13. Binjadhnan FAM, Ahmad T (2010) EEG Signals During Epileptic Seizure as a Semigroup of Upper Triangular Matrices. American J Applied Sciences 7:540-544

14. Chang HY, Yang SC, Lan SH, Chung PC (2010) Epileptic seizure detection in grouped multi-channel EEG signal using ICA and wavelet transform. In:

Proceedings of the IEEE International Symposium on Circuits and Systems, Paris, IEEE, Piscataway, pp 1388

15. De Clercq W, Lemmerling P, Van Paesschen W, Van Huffel S (2003) Characterization of interictal and ictal scalp EEG signals with the Hilbert transform. In: Proceedings of the 25th international conference of the IEEE Engineering Medicine Biology Society, Mexico, IEEE, Piscataway, pp 2459-2462

16. De ClercqW, Vergult A, Vanrumste B, Van Hees J, Palmini A, Van PaesschenW, Van Huffel S (2005) A new muscle artifact removal technique to improve the interpretation of the ictal scalp electroencephalogram. In: Proceedings of the 27th international conference of the IEEE Engineering Medicine Biology Society, Lyon, IEEE, Piscataway, pp 944-947

17. Dorr VL, Caparos M, Wendling F, Vignal J, Wolf D (2007) Extraction of reproducible seizure patterns based on EEG scalp correlations. Biomed signal processing and control 2:154-162

18. Fan J, Shao C, Ouyang Y, Wang J, Li S, Wang Z (2006) Automatic Seizure Detection Based on Support Vector Machines with Genetic Algorithms. In: Proceedings of SEAL, pp 845-852

19. Ghosh-Dastidar S, Adeli H, Dadmehr N (2007) Mixed-band wavelet chaos-neural network methodology for epilepsy and epileptic seizure detection. IEEE Trans Biomed Eng 54:1545-1551

20. Ghosh-Dastidar S, Adeli H, Dadmehr N (2008) Principal Component AnalysisEnhanced Cosine Radial Basis Function Neural Network for Robust Epilepsy and Seizure Detection. IEEE Trans Biomed Eng 55:512-518

21. Ghosh-Dastidar S, Adeli H (2009) A new supervised learning algorithm for multiple spiking neural networks with application in epilepsy and seizure detection. Neural Networks 22:1419-1431 
22. Gonzalez-Vellon B, Sanei S, Chambers JA (2003) Support vector machines for seizure detection. In: Proceedings of the 3rd international IEEE Symposium on Signal Processing and Information Technology, Darmstadt, IEEE, Piscataway, pp 126-129

23. Guarnizo C, Delgado E (2010) EEG single-channel seizure recognition using Empirical Mode Decomposition and normalized mutual information. In: Proceedings of the 10th international Conference on Signal Processing, Bejing, IEEE, Piscataway, pp 1-4

24. Guo L, Riveroa D, Pazosa A (2010) Epileptic seizure detection using multiwavelet transform based approximate entropy and artificial neural networks. J Neuroscience methods 193:156-163

25. Guo L, Rivero D, Dorado J, Rabunal JR, Pazos A (2010) Automatic epileptic seizure detection in EEGs based on line length feature and artificial neural networks. $\mathrm{J}$ Neuroscience Methods 19:101-109

26. Gupta D, James CJ (2007) Narrowband vs. Broadband Phase Synchronization Analysis Applied to Independent Components of Ictal and Interictal EEG. In: Proceedings of the international conference of the IEEE Engineering Medicine Biology Society, Lyon, IEEE, Piscataway, pp 3864-3867

27. Henriksen J, Remvig LS, Madsen RE, Conradsen I, Kjaer TW, Thomsen CE, Sorensen HBD (2010) Automatic seizure detection: going from sEEG to iEEG. In: Proceedings of the international conference of the IEEE Engineering Medicine Biology Society, Buenos Aires, IEEE, Piscataway, pp 2431-2434

28. Hesse CW, James CJ (2004) Stepwise model order estimation in blind source separation applied to ictal EEG. In: Proceedings of the 26th international conference of the IEEE Engineering Medicine Biology Society, California, IEEE, Piscataway, pp 986-989

29. Hesse CW, James CJ (2005) Tracking Epileptiform Activity in the Multichannel Ictal EEG using Spatially Constrained Independent Component Analysis. In: Proceedings of the 27th international conference of the IEEE Engineering Medicine Biology Society, Reading, IEEE, Piscataway, pp2067-2070

30. Hesse CW, James CJ (2007) Tracking and detection of epileptiform activity in multichannel ictal EEG using signal subspace correlation of seizure source scalp topographies. Med Biol Eng Comput 45:909-916

31. Hoeve M-J, Jones RD, Carroll GJ, Goelz H (2001) Automated detection of epileptic seizures in the EEG. In: Proceedings of the 23rd International Conference of the IEEE Engineering Medicine Biology Society, Istanbul, IEEE, Piscataway, pp 943946 
32. Hopfengartner R, Kerlinga F, Bauera V, Stefan H (2007) An efficient, robust and fast method for the offline detection of epileptic seizures in long-term scalp EEG recordings. Clin Neurophysiol 118:2332-2343

33. Hsu K, Yu S (2010) Detection of seizures in EEG using subband nonlinear parameters and genetic algorithm. Computers in Biology and Medicine 40:823-830

34. Jacquin A, Causevic E, John ER (2007). Automatic Identification of Spike-Wave Events and Non-Convulsive Seizures with a Reduced Set of Electrodes. In: Proceedings of the 29th international conference of the IEEE Engineering Medicine Biology Society, Lyon,IEEE, Piscataway, pp 1928-1931

35. James CJ, Demanuele C (2009) On spatio-temporal component selection in SpaceTime Independent Component Analysis: An application to ictal EEG. In: Proceedings of the International Conference of the IEEE Engineering Medicine Biology Society, Minnesota, IEEE, Piscataway, pp 3154-3157

36. James CJ, Hesse CW (2005) On the use of Spectrally Constrained ICA applied to single-channel Ictal EEG recordings within a Dynamical Embedding Framework. In: Proceedings of the 27th international conference of the IEEE Engineering Medicine Biology Society, Reading, IEEE, Piscataway, pp 956-959

37. James C, Lowe D (2000) Using dynamical embedding to isolate seizure components in the ictal EEG. In: Proceedings of the Science, Measurement and Technology, IEEE, Piscataway, pp 315-320

38. James CJ, Lowe D (2000) Using independent component analysis \& dynamical embedding to isolate seizure activity in the EEG. In: Proceedings of the 22nd international conference of the IEEE Engineering Medicine Biology Society, Chicago, IEEE, Piscataway, pp 1239-1332

39. Jing M, Sanei S (2007) Seizure signals separation using constrained topographic blind souce separation. In: Proceedings of the 15th European signal processing conference, Poznan, pp 1236-1240

40. Karakis I, Montouris GD, Otis JA, Douglass LM, Jonas R, Velez-Ruiz N, Wilforda $\mathrm{K}$, Espinosa PS (2010) A quick and reliable EEG montage for the detection of seizures in the critical care setting. J Clin Neurophysiol 27:100-105

41. Kelly KM, Shiaud DS, Kernd RT, Chiend JH, Yang MCK, Yandora KA, Valeriano JP, Halford JJ, Sackellares JC (2010) Assessment of a scalp EEG-based automated seizure detection system. Clin Neurphysiol 121:1832-1843

42. Khamis H, Mohamed A, Simpson S (2009) Seizure state detection of temporal lobe seizures by autoregressive spectral analysis of scalp EEG. Clin Neurophysiol 120:1479-1488 
43. Kim H, Rosen J (2010) Epileptic Seizure Detection - An AR Model Based Algorithm for Implantable Device. In: Proceedings of the international conference of the IEEE EngineeringMedicine Biology Society, Buenos Aires, IEEE, Piscataway, pp 5541

44. Kiymik MK, Subasi A, Ozcalik HR (2004) Neural networks with periodogram and autoregressive spectral analysis methods in detection of epileptic seizure. J Med Syst 28:511-522

45. Koessler L, Salido-Ruiz R, Ranta R, Louis-Dorr V, Gavaret M, Maillard L (2010) Influence of source separation and montage on ictal source localization. In: Proceedings of the international conference of the IEEE Engineering Medicine Biology Society, Buenos Aires, IEEE, Piscataway, pp 2898-2901

46. Kuhlmann L, Cook MJ, Fuller K, Grayden DB, Burkitt AN, Mareels IMY (2008) Correlation analysis of seizure detection features. In: Proceedings of the international conference Intelligent Sensors, Sensor Networks and Information Processing, Sydney, pp 309-314

47. Kuhlmann L, Burkitt AN, Cook MJ, Fuller K, Grayden DB, Seiderer L, Mareels IMY (2009) Seizure Detection Using Seizure Probability Estimation: Comparison of Features Used to Detect Seizures. Ann Biomed Eng 37:2129-2145

48. Li S, O’Brien T, Kiers L, Harley N, MacIssac C, Tan M, Carino J, Smit M, Strawhorn D, Bayley J, Day T (2010) The optimization of 2-channel EEG for the detection of generalized and temporal seizures: A pilot study for EEG monitoring study in the intensive care unit (CU). Clin Neurophysiol 121:e3

49. Liang S, Wang H, Chang W (2010) Combination of EEG complexity and spectral analysis for epilepsy diagnosis and seizure detection. $\mathrm{J}$ Advances in signal processing

50. McEvoy RP, Faul S, Marnane WP (2010) Ambulatory REACT: Real-time seizure detection with a DSP microprocessor. In: Proceedings of the international conference of the IEEE EngineeringMedicine Biology Society, Buenos Aires, IEEE, Piscataway, pp 2443-2446

51. McSharry PE, He T, Smith LA, Tarassenko L (2002) Linear and non-linear methods for automatic seizure detection in scalp electro-encephalogram recordings. Med Biol Eng Comput 40:447-461

52. Meier R, Dittrich H, Schulze-Bonhage A, Aertsen A (2008) Detecting Epileptic Seizures in Long-term Human EEG: A New Approach to Automatic Online and Real-Time Detection and Classification of Polymorphic Seizure Patterns. J Clin Neurophysiol 25:119-131 
53. Mirzaei A, Ayatollahi A, Gifani P, Salehi L (2010) EEG analysis based on waveletspectral entropy for epileptic seizures detection. In: Proceedings of the 3rd international conference on Biomedical Engineering and Informatics, Yantai, pp $878-882$

54. Mirzaei A, Ayatollahi A, Gifani P, Salehi L (2010) Spectral Entropy for Epileptic Seizures Detection. In: Proceedings of the 2nd international conference on computational intelligence, communication systems and networks, Liverpool, pp 301-307

55. Mohseni HR, Maghsoudi A, Shamsollahi MB (2006) Seizure Detection in EEG signals: A Comparison of Different approaches. In: Proceedings of the 28th international conference of the IEEE Engineering Medicine Biology Society, New York, IEEE, Piscataway, pp 6724-6727

56. Mohseni HR,Maghsoudi A, Kadbi MH, Hashemi J, Ashourvan A (2006) Automatc detection of epileptic seizure using time-frequency distributions. In: Proceedings of Advances in Medical, Signal and Informational Processing, Glasgow, Curran Associates

57. Mousavi SR, Niknazar M, Vahdat BV (2008) Epileptic Seizure Detection using AR Model on EEG Signals. In: Proceedings of the International Biomedical Engineering Conference, Singapore, pp 18-20

58. Minasyan GR, Chatten JB, Chatten MJ, Harner RN (2010) Patient-Specific Early Seizure Detection From Scalp Electroencephalogram. J Clin Neurophysiol 27:163178

59. Naghsh-Nilchi AR, Aghashahia M (2010) Epilepsy seizure detection using eigensystem spectral estimation andMultiple Layer Perceptron neural network. Biomedical signal processing and control 5:147-157

60. Pachori RB (2008) Discrimination between Ictal and Seizure-Free EEG Signals Using Empirical Mode Decomposition. Research Letters in Signal Processing

61. Pal PR, Panda R (2010) Classification of EEG signals for epileptic seizure evaluation. In: Proceedings of the IEEE Students' Technology Symposium, Kharagpur, IEEE, Piscataway, pp 72-76

62. Pravin Kumar S, Sriraam N, Benakop PG (2008) Automated detection of epileptic seizures using wavelet entropy feature with recurrent neural network classifier. In: Proceedings of the IEEE TENCON, Hyderabad, IEEE, Piscataway, pp 1-5

63. Pravin Kumar S, Sriraam N, Benakop, PG, Jinaga BC (2010) Entropies based detection of epileptic seizures with artificial neural network classifiers. Expert Sys with Applications 37:3284-3291 
64. Ramirez-Velez M, Staba R, Barth DS, Meyer FG (2006) Nonlinear classification of EEG data for seizure detection. In: Proceedings of the 3rd IEEE international symposium on Biomedical Imaging, Virginia, IEEE, Piscataway, pp 956-959

65. Sadati N,Mohseni HR,Maghsoudi A (2006) Epileptic Seizure Detection Using Neural Fuzzy Networks. In: Proceedings of the International Conference on Fuzzy Systems, Vancouver, IEEE, Piscataway, pp 596-600

66. Saleheen MU, Alemzadeh H, Cheriyan AM, Kalbarczyk Z, Iyer RK (2010) An efficient embedded hardware for high accuracy detection of epileptic seizures. In: Proceedings of the 3rd international conference on Biomedical Engineering and informatics, Yantai, pp 1889-1896

67. Sanei S, Latg MA, Shoker L (2004) Detection and localization of epileptic seizures using ICA. In: Proceedings of the IEEE Sensor Array and Multichannel signal processing workshop, Montreal, IEEE, Piscataway, pp 173-176

68. Schindler K, Wiest R, Kollar M, Donati F (2001) Using simulated neuronal cell models for detection of epileptic seizures in foramen ovale and scalp EEG. Clin Neurophysiol 112:1006-1017

69. Schneider M, Mustaro PN, Lima CAM (2009) Automatic recognition of epileptic seizure in EEG via support vector machine and dimension fractal. In: Proceedings of the International Joint Conference on Neural Networks, Atlanta, pp 3321-3325

70. Shiau D, Halford JJ, Kelly KM, Kern RT, Inman M, Chien J, Pardalos PM, Yang MCK, Sackellares JC (2010) Signal regularity-based automated seizure detection system for scalp EEG monitoring. Cybern Syst Anal 46:922-935

71. Slooter AJC, Vriens EM, Leijten FSS, Spijkstra JJ, Girbes ARJ, van Huffelen AC, Stam CJ (2006) Seizure detection in adult ICU patients based on changes in EEG synchronization likelihood. Neurocritical care 5:186-192

72. Song Y, Lio P (2010) A new approach for epileptic seizure detection: sample entropy based feature extraction and extreme learning machine. J Biomed Science and Eng 3:556-567

73. Stamoulis C, Chang BS (2009) Application of matched-filtering to extract EEG features and decouple signal contributions from multiple seizure foci in brain malformations. In: Proceedings of the 4th International IEEE EMBS Conference on Neural Engineering, Antalya, IEEE, Piscataway, pp 514-517

74. Subasi A (2005) Application of adaptive neuro-fuzzy inference system for epileptic seizure detection using wavelet feature extraction. Computers in Biology and Medicine 37:227-244

75. Subasi A (2005) Epileptic seizure detection using dynamic wavelet network. Expert Sys with Applications 29:343-355 
76. Subasi A (2006) Automatic detection of epileptic seizure using dynamic fuzzy neural networks. Expert Sys with Applications 31:320-328

77. Subasi A, Erc, elebi E, Alkan A, Koklukaya E (2006) Comparison of subspace-based methods with AR parametric methods in epileptic seizure detection. Computers in biology and medicine $36: 195-208$

78. Swiderski B, Osowski S, Rysz A (2005) Lyapunov exponent of EEG signal for epileptic seizure characterization. In: Proceedings of the European Conference on Circuit Theory and Design, Cork, pp 153-156

79. Tezela G, ozbay Y (2009) A new approach for epileptic seizure detection using adaptive neural network. Expert systems with applications 36:172-180

80. Tzallas AT, Tsipouras MG, Fotiadis DI (2007) A Time-Frequency Based Method for the Detection of Epileptic Seizures in EEG Recordings. In: Proceedings of the IEEE International Symposium on computer based medical systems, Maribor, IEEE, Piscataway, pp 135-140

81. Tzallas, AT, Tsipouras MG, Fotiadis DI (2007) The Use of Time-Frequency Distributions for Epileptic Seizure Detection in EEG Recordings. In: Proceedings of the international conference of the IEEE Engineering Medicine Biology Society, Lyon, IEEE, Piscataway, pp 3-6

82. Tzallas AT, Tsipouras MG, Fotiadis DI (2009) Epileptic seizure detection in EEGs using time-frequency analysis. IEEE Trans Information Technology 13:703-710

83. Ursino M, Magosso E, Gardella E, Rubboli G, Tassinari CA (2004) A wavelet based analysis of energy redistribution in scalp EEG during epileptic seizures. In: Proceedings of the 26th international conference of the IEEE Engineering Medicine Biology Society, California, IEEE, Piscataway, pp 255-258

84. Varsavsky A, Mareels I (2006) Patient Un-Specific Detection of Epileptic Seizures Through Changes in Variance. In: Proceedings of the 28th international conference of the IEEE Engineering Medicine Biology Society, New York,IEEE, Piscataway, pp 3747-3750

85. Varsavsky A, Mareels I (2007) A Complete Strategy for Patient Un-specific Detection of Epileptic Seizures Using Crude Estimations of Entropy. In: Proceedings of the 29th international conference of the IEEE Engineering Medicine Biology Society, Lyon, IEEE, Piscataway, pp 6491-6494

86. van Putten MJAM (2003) Nearest Neighbor Phase Synchronization as a Measure to Detect Seizure Activity from Scalp EEG Recordings. J Clin Neurophysiol 20:320325 
87. van Putten MJAM, Kind T, Visser F, Lagerburg V (2005) Detecting temporal lobe seizures from scalp EEG recordings: A comparison of various features. Clin Neurophysiol 116:2480-2489

88. Verma N, Shoeb A, Bohorquez J, Dawson J, Guttag J, Chandrakasan AP (2010). A Micro-Power EEG Acquisition SoC With Integrated Feature Extraction Processor for a Chronic Seizure Detection System. J Solid-State Circuits 45:804-816

89. Wilson SB, Scheuer ML, Emerson RG, Gabor AJ (2004) Seizure detection: evaluation of the Reveal algorithm. Clin Neurophysiol 115:2280-2291

90. Wilson SB (2006) Algorithm architectures for patient dependent seizure detection. Clin Neurophysiol 117:1204-1216

91. Wongsawat Y (2008) Epileptic Seizure Detection in EEG Recordings using Phase Congruency. In: Proceedings of the 30th international conference of the IEEE Engineering Medicine Biology Society, Vancouver, IEEE, Piscataway, pp 927-930

92. Xie S, Lawniczak AT, Song Y, Lio P (2010) Feature extraction via dynamic PCA for epilepsy diagnosis and epileptic seizure detection. In: Proceedings of the IEEE international workshop on Machine Learning for signal processing, Kitila, IEEE, Piscataway, pp 337-342

93. Yuan Y (2010) Detection of Epileptic Seizure Based on EEG Signals. In: Proceedings of the 3rd International Congress on Image and signal processing, Quebec, pp 4209-4211

94. Yuan Y, Li Y, Yu D, Mandic DP (2008) Automated Detection of Epileptic Seizure Using Artificial Neural Network. In: Proceedings of the 2nd International Conference on Bioinformatics and Biomedical Engineering, Shanghai, pp 1959-1962

95. Zandi AS, Tafreshi R, Dumont GA, Ries CR, MacLeod BA, Puil E (2007) Electroconvulsive Therapy: A Model for Seizure Detection by a Wavelet Packet Algorithm. In: Proceedings of the 29th International Conference of the IEEE Engineering Medicine Biology Society, Lyon, IEEE, Piscataway, pp 1916-1919

96. Zandi AS, Dumont GA, Javidan M, Tafreshi R, MacLeod BA, Ries CR, Puil E (2008) A novel wavelet based index to detect epileptic seizures using scalp EEG signals. In: Proceedings of the 30th International Conference of the IEEE Engineering Medicine Biology Society, Vancouver, IEEE, Piscataway, pp 919-922

97. Zandi AS, Javidan M, Dumont GA, Tafreshi R (2010) Automated real-time epileptic seizure detection in scalp EEG recordings using an algorithm based on wavelet packet transform. IEEE Trans Biomed Eng 57:1639-1651 


\subsection{Algorithm segmentation}

Based upon these 97 publications, the process of seizure detection is broken down here into three principal stages: signal conditioning and pre-processing to condition the recorded EEG signal; feature extraction where the characteristic features of the EEG trace are calculated; and post-processing and classification where these features are combined and used to make a decision as to whether a seizure is present in the current EEG. The initial two stages are now considered in turn, and in particular the pre-processing methods evaluated in terms of their popularity and possible low power hardware implementation. This is necessary as low computational complexity features, which rely on power intensive pre-processing are of course not suitable for consideration.

\subsection{Signal conditioning and pre-processing}

Pre-processing methods reported in more than one of the 97 publications studied have been summarized in Table A1 below. It is clear that basic filtering techniques (low pass and band pass in particular) are exceptionally popular. This is mainly due to the presence of high frequency artefacts in scalp EEG signals, and restricting the frequencies present to those principally of interest for epileptiform activity $(0-25 \mathrm{~Hz})$ can help alleviate their impact. High pass and notch filters are less common, although such filters are often incorporated into the EEG recording hardware itself. Indeed the International Federation of Clinical Neurophysiology recommends high pass filtering the signal with a cut-off frequency of less than or equal to $0.16 \mathrm{~Hz}$ to remove any dc offset.

It is also clear that other spectral estimation methods, artefact rejection techniques and blind source separation have also been popular, although these categories encompass a wide variety of techniques that have been grouped together here. In contrast, the Discrete Wavelet Transform (DWT) and the Fourier Transform (FT) are also popular and more specific. These transforms, in addition to the Continuous Wavelet Transform (CWT), have been previously implemented as low power dedicated circuits as discussed in the main text. Features extracted after applying each of these pre-processing methods, in addition to features derived from the time domain signal (which requires no preprocessing), are discussed in Section 2. 
Table A1 Signal conditioning and pre-processing methods used in more than one of the 97 publications considered.

\begin{tabular}{|c|c|c|}
\hline Category & Method & Number of papers \\
\hline \multirow[t]{8}{*}{ Signal conditioning } & Artefact rejection & 10 \\
\hline & Band pass filter & 27 \\
\hline & High pass filter & 4 \\
\hline & Low pass filter & 23 \\
\hline & Mean correction & 2 \\
\hline & Notch/band stop filter & 4 \\
\hline & Re-montaging & 4 \\
\hline & Windowing & 2 \\
\hline \multirow[t]{6}{*}{ Transforms } & Continuous Wavelet Transform (CWT) & 4 \\
\hline & Discrete Wavelet Transform (DWT) & 21 \\
\hline & Fourier Transform (FT) & 13 \\
\hline & Hilbert Transform & 2 \\
\hline & Wavelet Packet Transform & 4 \\
\hline & Other spectral estimation methods & 13 \\
\hline \multirow[t]{3}{*}{ Others } & Auto-regressive modelling & 8 \\
\hline & Blind source separation & 12 \\
\hline & Empirical mode decomposition & 2 \\
\hline
\end{tabular}




\section{Features implemented in this study}

From the 97 short-listed adult, scalp EEG seizure detection publications, a total of 65 features derived from four pre-processing methods - time domain, Discrete Wavelet Transform (DWT), Continuous Wavelet Transform (CWT) and Fourier Transform (FT) were selected for investigation in terms of their detection performance (sensitivity, specificity, area under the sensitivity-specificity trade-off curve) and relative complexity. Full details on the calculations used to generate each of these features are given below. All features are calculated on non-overlapping $2 \mathrm{~s}$ epochs of EEG data.

\subsection{Time domain features}

These features are calculated on each non-overlapping epoch of the raw input EEG signal. Here the input signal is denoted $x(n)$ where $n$ is the sample number in time. $N$ is the total number of samples in an epoch.

- Complexity $[2,50,59,90]$ and defined here as

$$
F=\frac{\sigma_{x^{\prime \prime}}}{\sigma_{x^{\prime}}} / \frac{\sigma_{x^{\prime}}}{\sigma_{x}}
$$

where $\sigma$ denotes the standard deviation of the signal $x, x^{\prime}$ is the first derivative of the input signal and $x^{\prime \prime}$ is the second derivative.

- Energy/power $[52,61,81,84]$ and defined here as

$$
F=\frac{1}{N} \sum_{n=1}^{N} x(n)^{2}
$$

- Fractal dimension $[2,58,69]$ and defined here using Katz's algorithm as

$$
F=\frac{\log _{10} L}{\log _{10} d}
$$

where $d$ is the maximum of all points $x(n)$ minus the starting value $x(1)$ within an epoch. $L$ is the absolute sum of distances between adjacent points.

- Kurtosis $[10,50,61]$ and defined here as

$$
F=\frac{1}{N} \sum_{n=1}^{N}\left(x(n)-\mu_{x}\right)^{4} /\left\{\frac{1}{N} \sum_{n=1}^{N}\left(x(n)-\mu_{x}\right)^{2}\right\}^{2}-3,
$$

where $\mu_{x}$ is the mean of the input signal within the epoch. 
- Line length $[43,50]$ and defined here as

$$
F=\sum_{n=2}^{N}|x(n-1)-x(n)|
$$

- Maximum $[70,79]$ and defined here as the largest value within an epoch

$$
F=\max (x(n))
$$

- Mean $[79,87]$ and defined here as

$$
F=\frac{1}{N} \sum_{n=1}^{N} x(n)
$$

- Minimum $[70,79]$ and defined here as the smallest value of $x(n)$ within an epoch

$$
F=\min (x(n))
$$

- Mobility $[2,50,90]$ and defined here as

$$
F=\frac{\sigma_{x^{\prime}}}{\sigma_{x}}
$$

where $\sigma$ denotes the standard deviation of the signal $x$ and $x^{\prime}$ is the first derivative of the input signal.

- Non-linear energy $[50,58]$ and defined here as

$$
F=\frac{1}{N-2} \sum_{n=2}^{N-1} x(n)^{2}-x(n+1) x(n-1)
$$

- Relative derivative [46, 47] and defined here by initially calculating the absolute derivative within the epoch

$$
g(n)=\left|\frac{x(n)-x(n-1)}{\Delta n}\right|
$$

where

$$
\Delta n=\frac{1}{\text { Sampling frequency }}
$$

To generate the feature $F$ the mean of the absolute derivative $g(n)$ within the current epoch is divided by the standard deviation of $g(n)$ over $30 \mathrm{~s}$ ending 1 minute prior to last epoch. 
- Shannon entropy $[8,10,50,61,85]$ and defined here as

$$
F=-\sum_{n=0}^{N-1} p_{n}(x)^{2} \log _{2}\left(p_{n}(x)\right)^{2}
$$

where $p_{n}(x)$ is the probability of $x(n)$ occurring within an epoch of $N$ values. For example, if $x(n)=\{1,1,3,5,6 \ldots\}$ then the probability of 1 occurring is $2 / N$.

- Skewness $[10,50]$ and defined here as

$$
F=\frac{1}{N} \sum_{n=1}^{N}\left(x(n)-\mu_{x}\right)^{3} /\left\{\frac{1}{N} \sum_{n=1}^{N}\left(x(n)-\mu_{x}\right)^{2}\right\}^{3 / 2}
$$

where $\mu_{x}$ is the mean of the input signal within the epoch.

- Total minima and maxima $[50,87]$ and defined here as $\mathrm{F}$ is the total number of local maxima and minima within an epoch. A local maxima or minima occurs when

$$
\frac{d x(n)}{d n}<0.01
$$

- Variance/standard deviation $[2,10,32,41,50-52,55,59,66,70,79,90]$ and variance has been defined here as

$$
F=\frac{1}{N} \sum_{n=1}^{N}\left(x(n)-\mu_{x}\right)^{2}
$$

where $\mu_{x}$ is the mean of the input signal within the epoch. The standard deviation is the square root of this.

- Zero crossing $[41,50,70,87]$ and calculated here by initially subtracting the mean of the input EEG $x(n)$ within an epoch

$$
y(n)=x(n)-\mu_{x}
$$

The feature $F$ is then given by the total number of positive going zero crossings within the epoch where a positive going zero crossing is defined as

$$
y(n)\langle\varepsilon \text { and } y(n+5)\rangle \varepsilon
$$

where $\varepsilon=0.01 \mu \mathrm{V}$.

- Zero crossing of first derivative $[50,52]$ and defined here as above for the zero crossing, but calculated on the first derivative of the input signal $x^{\prime}$. 


\subsection{Discrete Wavelet Transform-based features}

The following features are calculated using the Discrete Wavelet Transform (DWT) (MATLAB built-in function) with the commonly used Daubechies-4 wavelet. The features are computed only for frequencies below $25 \mathrm{~Hz}$ to match the frequencies of interest for epileptiform activity. To do this, the input EEG signal $x(n)$ is split into nonoverlapping epochs of $N$ samples and each epoch is decomposed into five levels of detail coefficients and one level approximation coefficient covering the frequency ranges

- D3: $12.5-25 \mathrm{~Hz}$.

- D4: $6.25-12.5 \mathrm{~Hz}$.

- D5: $3.125-6.25 \mathrm{~Hz}$.

- A5: $0.16-3.125 \mathrm{~Hz}$.

Each of the basic features defined below is calculated separately in each one of these frequency bands.

The feature definitions below use the terminology that there are $M$ frequency ranges $(M=4)$ and current frequency range is $i$. At each frequency range, a total of $K$ wavelet coefficients have been produced with $k$ indexing a particular coefficient.

- Bounded variation $[46,47]$ and defined here as

$$
F_{i}=\frac{\sum_{k=2}^{K}\left|D_{i}(k)-D_{i}(k-1)\right|}{\max _{k} D_{i}-\min _{k} D_{i}}
$$

- Coefficients [58, 74-76] and defined here as

$$
F_{i}=\frac{1}{K} \sum_{k=1}^{K} D_{i}(k)
$$

- Energy $[50,55,65,74,83]$ and defined here as

$$
F_{i}=\frac{1}{K} \sum_{k=1}^{K} D_{i}(k)^{2}
$$

- Entropy $[53,54,58,63]$ and defined here as

$$
F_{i}=-\sum_{k=1}^{K} D_{i}(k)^{2} \log _{2}\left(D_{i}(k)^{2}\right)
$$

- Relative bounded variation [46, 47] and defined here such that the bounded variation in the current epoch defined above, and the feature $F_{i}$ is generated by dividing this by the mean of the bounded variation over a $30 \mathrm{~s}$ period ending 1 minute prior to the last epoch. 
- Relative power $[46,47]$ and defined here by first calculating the foreground power in the current epoch $e$

$$
F G_{i}(e)=\operatorname{median}\left\{D_{i}^{2}\right\}
$$

The background power is then the foreground power tracked over the past 120 epochs using a decaying memory with constant $\lambda=0.99923$

$$
B G_{i}(e)=(1-\lambda) \operatorname{median}\left\{F G_{i}(e-1) \ldots F G_{i}(e-120)\right\}+\lambda B G_{i}(e-1)
$$

Finally the relative power is the ratio of foreground to background power

$$
F_{i}=\frac{F G_{i}(e)}{B G_{i}(e)}
$$

- Relative scale energy $[46,47]$ and defined here by initially calculating the energy in frequency band $\mathrm{i}$

$$
E_{i}=\sum_{k=1}^{K} D_{i}(k)^{2} \frac{N}{K}
$$

where $N$ is the number of samples in an epoch. The relative scale energy is then calculated as the energy in frequency band $i$ as a fraction of the total energy in the 0.16$25 \mathrm{~Hz}$ frequency range

$$
F_{i}=\frac{E_{i}}{\sum_{i=1}^{M} E_{i}}
$$

- Variance/standard deviation $[19-21,58,74]$ and variance is defined here as

$$
F_{i}=\frac{1}{K} \sum_{k=1}^{K}\left(D_{i}(k)-\mu_{D}\right)^{2}
$$

where $\mu_{D}$ is the mean of the wavelet coefficients in the frequency range $i$. The standard deviation is the square root of this. 


\subsection{Continuous Wavelet Transform-based features}

The following features are calculated based upon the Continuous Wavelet Transform (CWT) (MATLAB built-in function) of each non-overlapping epoch of the input EEG signal $x(n)$. The CWT mother wavelet and analysis scale/frequency are defined for each feature separately below. Here the CWT coefficients are denoted as $C_{k}$ where $k$ is the coefficient index and there are a total of $N$ coefficients at each analysis frequency.

- Coefficient z-score [9] and defined here by initially calculating the CWT using the Symlet5 wavelet centred at $4.2 \mathrm{~Hz}$. The coefficient z-score is then calculated as

$$
F=\frac{1}{N} \sum_{k=1}^{N} \frac{C_{k}-\mu_{C}}{\sigma_{C}}
$$

where $\mu_{C}$ is the mean of the CWT coefficients and $\sigma_{C}$ is their standard deviation.

- Energy $[9,52]$ and is calculated here using the Symlet5 CWT centred at $4.2 \mathrm{~Hz}$ as

$$
F=\frac{1}{N} \sum_{k=1}^{N} C_{k}^{2}
$$

- Entropy [62] and calculated here using the Mexican hat CWT centred at frequency $j$.

Firstly the Mexican hat CWT is performed at $S$ scales/centre frequencies, with the centre frequencies spanning the frequency band $i$ (the same frequency bands used with the DWT-based features.) This is used to give the energy in the frequency band $i$

$$
E_{i}=\sum_{j=1}^{S} \sum_{k=1}^{N}\left|C_{j k}\right|^{2}
$$

Then the Mexican hat CWT is performed with a total of T scales covering the $0.5-50 \mathrm{~Hz}$ frequency band and the sum of the energy across all frequency ranges $i$ found

$$
E_{T}=\sum_{i=1}^{T} E_{i}
$$

Finally, the entropy is calculated as

$$
F=-\sum_{i=1}^{T} \frac{E_{i}}{E_{T}} \log _{2} \frac{E_{i}}{E_{T}}
$$

- Standard deviation of energy [9] and calculated here using Symlet5 CWT centred at $4.2 \mathrm{~Hz}$. The feature $F$ is computed as the standard deviation of the coefficients $C_{k}$. 


\subsection{Fourier Transform-based features}

The following features are calculated based upon the Fourier Transform (FT) of the input EEG signal $x(n)$. In all cases the FT is obtained by applying a 512-point Fast Fourier Transform (FFT) (MATLAB built-in function) to each epoch of the raw EEG data. Here $S_{m}$ are the Fourier coefficients in frequency bin $m$, and the spectral power in frequency bin $m$ is denoted by $p_{m}$ and given by $S_{m}{ }^{2}$.

- Median frequency $[2,55]$ and defined here as

$$
F=\arg \left\{\underset{m}{\operatorname{median}}\left(p_{m}\right)\right\}
$$

That is, the frequency at which the power $p_{m}$ is approximately equal to $50 \%$ of the maximum power.

- Peak frequency $[50,58,61]$ and defined here as the frequency at which the maximum power occurs

$$
F=\arg \left\{\max _{m}\left(p_{m}\right)\right\}
$$

- Power $[32,49,50,90]$ and defined here as

$$
F_{i}=\sum p_{m}
$$

where $p_{m}$ is summed into the four different frequency ranges $i$, as used with the DWT features above.

- Spectral edge frequency $[50,90]$ and defined here as the frequency below which $90 \%$ of the total spectral power in the $2-20 \mathrm{~Hz}$ frequency band lies.

- Spectral entropy $[2,50,54,55,58,62,63]$ and defined here as

$$
F_{i}=-\sum p_{m} \log _{2} p_{m}
$$

where $p_{m}$ is summed for the four different frequency ranges $i$, as used with the DWT features above.

- Total spectral power $[50,55,58]$ and defined here as the sum of $p_{m}$ within the $2-20 \mathrm{~Hz}$ region. 


\section{Test database}

Features are compared using adult scalp EEG signals with a total duration over 172 hours from 24 adults with a total of 47 seizures marked by medical experts for seizure start and end. The data was recorded during routine, ambulatory and long-term monitoring at the National Society of Epilepsy (UK), Katholieke Universiteit Leuven (Belgium) and Freiburg University Hospital (Germany). Records analysed have been selected to not contain any discontinuities. These records were not marked for interictal events and have not been screened to remove artefact prone recordings. Details of the records present in the test database have been listed in Table A2. Here, all records that contain an expert marked seizure also contain non-seizure sections (or epochs), and there is only a single seizure in each seizure record.

All data has been sampled at or above $200 \mathrm{~Hz}$ and has been re-sampled to $200 \mathrm{~Hz}$ prior to testing. Features are tested using the 16 channels common to all records: C3, C4, CZ, F3, F4, FZ, F7, F8, FP1, FP2, O1, O2, T3, T4, T5 and T6. Per channel, there are $26982 \mathrm{~s}$ seizure epochs and 308630 non-seizure epochs present. Non-seizure epochs include background data and may include pre-ictal data, post-ictal data, interictal spikes and/or artefacts, as every epoch within a record that is not classified as a seizure epoch is included in this category. Artefact-prone data and sections likely to contain pre-ictal and post-ictal discharges have not been removed from the test database, in order to test the performance of the features on data similar to what may be expected in a clinical or ambulatory monitoring session. It should also be noted that as the data is divided into non-overlapping epochs, there are special cases where an epoch contains the start or end of an expert marked seizure in addition to non-seizure data. These are also classified as seizure epochs, as such epochs are deemed to contain ictal information which is of medical interest. 
Table A2 Details of records present in the test database

\begin{tabular}{|c|c|c|c|c|c|c|c|}
\hline \multirow[t]{2}{*}{ Subject } & \multirow[t]{2}{*}{ Age } & \multirow[t]{2}{*}{ Sex } & \multirow{2}{*}{$\begin{array}{l}\text { Total recording } \\
\text { duration } \\
\text { (HH:MM:SS) }\end{array}$} & \multicolumn{2}{|c|}{ Seizure } & \multicolumn{2}{|c|}{ Non-seizure } \\
\hline & & & & $\begin{array}{l}\text { Records / } \\
\text { Events }\end{array}$ & Epochs & Records & Epochs \\
\hline 1 & 53 & $\mathrm{~F}$ & $02: 20: 50$ & 1 & 59 & 1 & 4167 \\
\hline 2 & 22 & $\mathrm{~F}$ & $00: 05: 10$ & 1 & 4 & 0 & 151 \\
\hline 3 & 33 & $\mathrm{~F}$ & $34: 02: 06$ & 3 & 148 & 39 & 61126 \\
\hline 4 & 56 & $\mathrm{~F}$ & $66: 59: 37$ & 7 & 417 & 78 & 120195 \\
\hline 5 & 41 & $\mathrm{~F}$ & $21: 26: 31$ & 7 & 1168 & 16 & 37430 \\
\hline 6 & 35 & $\mathrm{M}$ & $10: 45: 20$ & 1 & 38 & 0 & 19322 \\
\hline 7 & 35 & $\mathrm{M}$ & $12: 04: 50$ & 1 & 51 & 0 & 21694 \\
\hline 8 & 28 & $\mathrm{~F}$ & 00:10:59 & 2 & 44 & 0 & 286 \\
\hline 9 & 60 & $\mathrm{M}$ & $03: 31: 13$ & 2 & 118 & 1 & 6220 \\
\hline 10 & 33 & $\mathrm{M}$ & 04:08:57 & 1 & 49 & 2 & 7421 \\
\hline 11 & 23 & $\mathrm{~F}$ & $01: 14: 10$ & 2 & 26 & 0 & 2200 \\
\hline 12 & 34 & $\mathrm{M}$ & $02: 05: 27$ & 1 & 8 & 1 & 3756 \\
\hline 13 & - & $\mathrm{M}$ & 04:16:07 & 3 & 116 & 2 & 7569 \\
\hline 14 & 22 & $\mathrm{~F}$ & $00: 49: 59$ & 1 & 48 & 1 & 1452 \\
\hline 15 & 35 & $\mathrm{~F}$ & $00: 31: 55$ & 6 & 58 & 0 & 901 \\
\hline 16 & 46 & $\mathrm{~F}$ & 00:06:12 & 1 & 75 & 0 & 111 \\
\hline 17 & - & - & $00: 26: 42$ & 3 & 51 & 0 & 751 \\
\hline 18 & - & $\mathrm{F}$ & $00: 38: 36$ & 3 & 54 & 0 & 1105 \\
\hline 19 & - & $\mathrm{M}$ & 00:09:05 & 1 & 166 & 0 & 107 \\
\hline 20 & 47 & $\mathrm{~F}$ & 02:00:11 & 0 & 0 & 1 & 3606 \\
\hline 21 & 45 & $\mathrm{~F}$ & $00: 46: 19$ & 0 & 0 & 2 & 1390 \\
\hline 22 & 43 & $\mathrm{M}$ & $00: 15: 15$ & 0 & 0 & 1 & 458 \\
\hline 23 & 47 & $\mathrm{M}$ & 02:00:11 & 0 & 0 & 1 & 3606 \\
\hline 24 & 28 & $\mathrm{~F}$ & $02: 00: 11$ & 0 & 0 & 1 & 3606 \\
\hline & & Total & $172: 55: 53$ & 47 & 2698 & 147 & 308630 \\
\hline
\end{tabular}

